\title{
Saúde bucal na rede de atenção e processo de regionalização
}

\author{
Oral health care in the health network \\ and the regionalization process
}

Ana Lúcia Schaefer Ferreira de Mello ${ }^{1}$

Selma Regina de Andrade ${ }^{2}$

Samuel Jorge Moysés ${ }^{3}$

Alacoque Lorenzini Erdmann ${ }^{2}$

${ }^{1}$ Departamento de

Odontologia, Universidade

Federal de Santa Catarina.

Campus Universitário João

David Ferreira Lima,

Trindade. 88.040-270

Florianópolis SC Brasil.

alfm@terra.com.br

${ }^{2}$ Departamento de

Enfermagem, Universidade

Federal de Santa Catarina.

${ }^{3}$ Escola de Saúde e

Biociências, Pontifícia

Universidade Católica do

Paraná.
Abstract The scope of this article is to outline the construction of the regionalized health network, focusing on oral health care and identifying the problems and the progress in its implementation. The context was the proposed national network model in the policies that underpin the Unified Health System. This is a descriptive-exploratory study with a qualitative approach methodologically supported by Grounded Theory. Interviews were conducted with managers, health professionals and teachers graduated in Dentistry, forming three sample groups. The core category was named "Enhancing the interaction of oral health care in the health network in the regionalization process." In the proposed theoretical model, the enhancement of interaction arises from conditions such as: the characterization of oral health care in the regionalized health network; the identification of difficulties in the implementation process; and the analysis of the operation of the oral health care network. The inclusion of oral health in the network is at an early stage, sometimes detached from the set of relations that constitutes the health network, which is also being consolidated. Evidence gathered in this study indicates the structuring of the regionalized health care network as a prerequisite to raise oral health care and assistance to a new level.

Key words Regionalization, Oral health, Health care
Resumo O artigo tem por objetivo caracterizar a construção da rede regionalizada de saúde, focalizando a atenção à saúde bucal e identificando dificuldades e avanços na sua implementação. Teve por contexto o modelo de rede sugerido nacionalmente pelas políticas que orientam o Sistema Único de Saúde. Estudo exploratório-descritivo, com abordagem qualitativa e sustentação metodológica na Teoria Fundamentada nos Dados. Realizaram-se entrevistas com gestores, profissionais de saúde e professores graduados em Odontologia, constituindo três grupos intencionais. A categoria central denominou-se "Potencializando a interação no cuidado à saúde bucal na rede de atenção à saúde no processo de regionalização". No modelo teórico proposto, a potencialização das interações decorre de condições como: Caracterização do cuidado à saúde bucal na rede regionalizada de atenção à saúde; Identificação das dificuldades na implementação e Análise do funcionamento da rede de atenção à saúde bucal. A inserção da saúde bucal na rede está no início, por vezes descolada do conjunto de relações que constitui a rede de atenção que, também, está em consolidação. Os elementos recolhidos neste estudo indicam a implantação da rede regionalizada de atenção como condição para levar o cuidado à saúde bucal a um novo patamar de atenção e assistência.

Palavras-chave Regionalização, Saúde bucal, Assistência à saúde 


\section{Introdução}

A descentralização e a regionalização das ações e serviços de saúde no Brasil são diretrizes estruturantes na conformação do Sistema Único de Saúde (SUS). As competências executivas foram transferidas, em larga escala, da esfera federal à estadual e destas, aos gestores municipais, porém desacompanhadas, em sua plenitude, de autonomia financeira, melhorias na capacidade de gestão e integração das distintas dimensões da atenção à saúde ${ }^{1-3}$. A integração da diversidade de ações e serviços, exigida para atender as necessidades de saúde da população, implica um arranjo organizacional sob forma de rede, cujo desafio reside no desenvolvimento de relações horizontais entre os pontos de atenção que se encontram articulados, sob regência da Atenção Primária de Saúde (APS) ${ }^{4-7}$.

A resolução das dificuldades desencadeadas no processo de descentralização, principalmente quanto à capacidade dos municípios proverem o cuidado à saúde, incluindo a bucal, de forma integral, resolutiva e capaz de atender adequadamente às demandas sociais, passa pela conformação de redes de serviços integradas e regionalizadas. Estas redes deveriam estar acompanhadas de dispositivos institucionalizados de planejamento, programação e regulação, garantindo, de forma mais racional, o acesso da população a todos os níveis de atenção ${ }^{8}$. Com base neste pressuposto, o governo brasileiro propôs a (re)estruturação do sistema de saúde, na perspectiva de Rede de Atenção, como estratégia de superação do modo fragmentado de operar a atenção e a gestão nas distintas Regiões de Saú$\mathrm{de}^{9}$. Rede de Atenção à Saúde é entendida como decorrente de "arranjos organizativos de ações e serviços de saúde, de diferentes densidades tecnológicas, que integradas por meio de sistemas de apoio técnico, logístico e de gestão, buscam garantir a integralidade do cuidado"'.

No âmbito da saúde bucal, o serviço público dá sinais de realinhamento conceitual e operativo, rompendo com o paradigma "odontocentrado" e a atitude corporativa isolacionista. Destacam-se as experiências de inserção na Estratégia Saúde da Família (ESF), de regulação e ampliação de acesso a linhas de cuidado e níveis tecnológicos, que incluem os Centros de Especialidades Odontológicas (CEO), bem como novas bases para a formação profissional ${ }^{10}$. Tais decisões conduzem a mudanças que consolidam as diretrizes da Política Nacional de Saúde Bucal ${ }^{11}$. O modelo de atenção em saúde bucal, organiza- do em rede, provavelmente opera com melhor capacidade de resposta às aspirações e às necessidades individuais e coletivas, compensando a fracionada e insuficiente presença histórica do Estado no campo da saúde bucal coletiva.

Os pressupostos que regem a reorientação do modelo de atenção em saúde bucal, no Brasil, incluem integralidade, resolutividade e qualificação da atenção primária, indissociavelmente articulada ao conjunto da rede de serviços ${ }^{3}$. Numa perspectiva de assistência individual, os marcos legais indicam a constituição de uma rede progressiva de cuidados à saúde bucal, com o estabelecimento dos fluxos de referência e contrarreferência para as diversas especialidades. De outra parte, numa abordagem coletiva e de prevenção dos agravos em saúde bucal, recomendam o investimento em ações intersetoriais, tal como acesso a água tratada e fluoretada, bem como o enfrentamento das iniquidades sanitárias decorrentes da determinação social do processo saúdedoença ${ }^{11}$. Contudo, grandes lacunas e insuficiências organizativas ainda são observáveis, especialmente no contexto de grandes aglomerados urbanos, onde os problemas de integração de variados serviços se adensam, devido ao acúmulo histórico de distintas culturas institucionais e complexas demandas populacionais.

Nesse contexto, o propósito deste estudo é caracterizar a construção da rede regionalizada de saúde, focalizando a atenção à saúde bucal e identificando dificuldades e avanços no seu processo de implementação. Teve por contexto o modelo de rede sugerido nacionalmente pelas políticas que orientam o Sistema Único de Saúde (SUS).

\section{Método}

Trata-se de estudo exploratório-descritivo, com utilização de técnicas qualitativas de pesquisa tanto para a coleta, quanto para a análise dos dados. A análise qualitativa correspondeu a um processo de interpretação dos dados brutos e organização de um modelo teórico. Esse procedimento seguiu a base teórico-metodológica denominada Teoria Fundamentada nos Dados $(\mathrm{TFD})^{12,13}$.

A pesquisa foi realizada na Região de Saúde da Grande Florianópolis, Santa Catarina, Brasil. Dentre os critérios mínimos de assistência ambulatorial e hospitalar que delimitam uma Região de Saúde, constam como obrigatórios os grupos de diagnóstico, consulta médica especi- 
alizada e os procedimentos de odontologia. Com relação a estes últimos, cada região de saúde deve dispor de um Centro de Especialidades Odontológicas (CEO), que seja referência para, no mínimo, três municípios.

A Região estudada é composta por 22 municípios, com uma população de 1.027.280 habitantes $^{14}$. Os equipamentos e serviços de saúde estão concentrados, majoritariamente, em dois municípios: Florianópolis e São José. No âmbito do SUS, conta com 189 Centros de Saúde, 16 Policlínicas/Ambulatórios especializados, 4 serviços de Pronto Atendimento e 53 Hospitais (9 hospitais-dia, 14 hospitais especializados e 30 hospitais gerais). Atuavam, em 2011, 254 equipes de saúde: 49,2\% equipes de Saúde da Família (ESF) (sem equipes de saúde bucal - ESB) e 50,7\% ESF com ESB (93\% tipo I); as demais, equipes de apoio e de agentes comunitários de saúde. $\mathrm{Na}$ média complexidade odontológica, existem atualmente 6 CEO (1 CEO: 168.800 habitantes), sendo um deles localizado na Universidade Federal de Santa Catarina (UFSC) ${ }^{14}$. Em Florianópolis, as duas Unidades de Pronto Atendimento (UPA) possuem atendimento odontológico 24 horas.

A seleção dos participantes, de caráter intencional, considerou como critérios de inclusão: formação na área de Odontologia e atuação nos âmbitos de formação, prestação de serviços e/ou gestão do sistema público de saúde, no contexto estudado. No total, foram entrevistadas quinze pessoas. Os critérios utilizados na TFD para apreensão teórica das dimensões e construtos da realidade investigada nortearam a coleta e a análise do material, tanto para definição do número de participantes, como a inclusão de novos grupos de sujeitos (Figura 1). Este procedimento é denominado Amostragem Teórica e estabelece os Grupos Amostrais ${ }^{12,13}$. Assim, o primeiro Grupo Amostral foi composto por cinco profissionais de saúde no exercício de atividades no SUS, na atenção primária e secundária (média complexidade); o Segundo Grupo Amostral composto por cinco gestores do SUS, dos níveis estadual e municipal, especificamente no âmbito da saúde bucal; e o Terceiro Grupo Amostral com cinco professores do Curso de Odontologia da UFSC, com experiência em gestão e integração ensino-serviço. As entrevistas foram realizadas durante os meses de maio, junho e julho de 2009.

O material obtido com as entrevistas foi transcrito e analisado com o auxílio do software NVivo $8.0^{\circledR}$. Na TFD, a fase de coleta de dados se superpõe a de análise, concomitantemente, seguindo-se as etapas de codificação aberta, axial e seletiva do material empírico ${ }^{12}$. Os códigos gerados foram agrupados em categorias por meio da Análise Comparativa dos dados ${ }^{13}$. Nesta técnica, os elementos de análise fundamentam novas hipóteses sob as relações entre categorias obtidas e

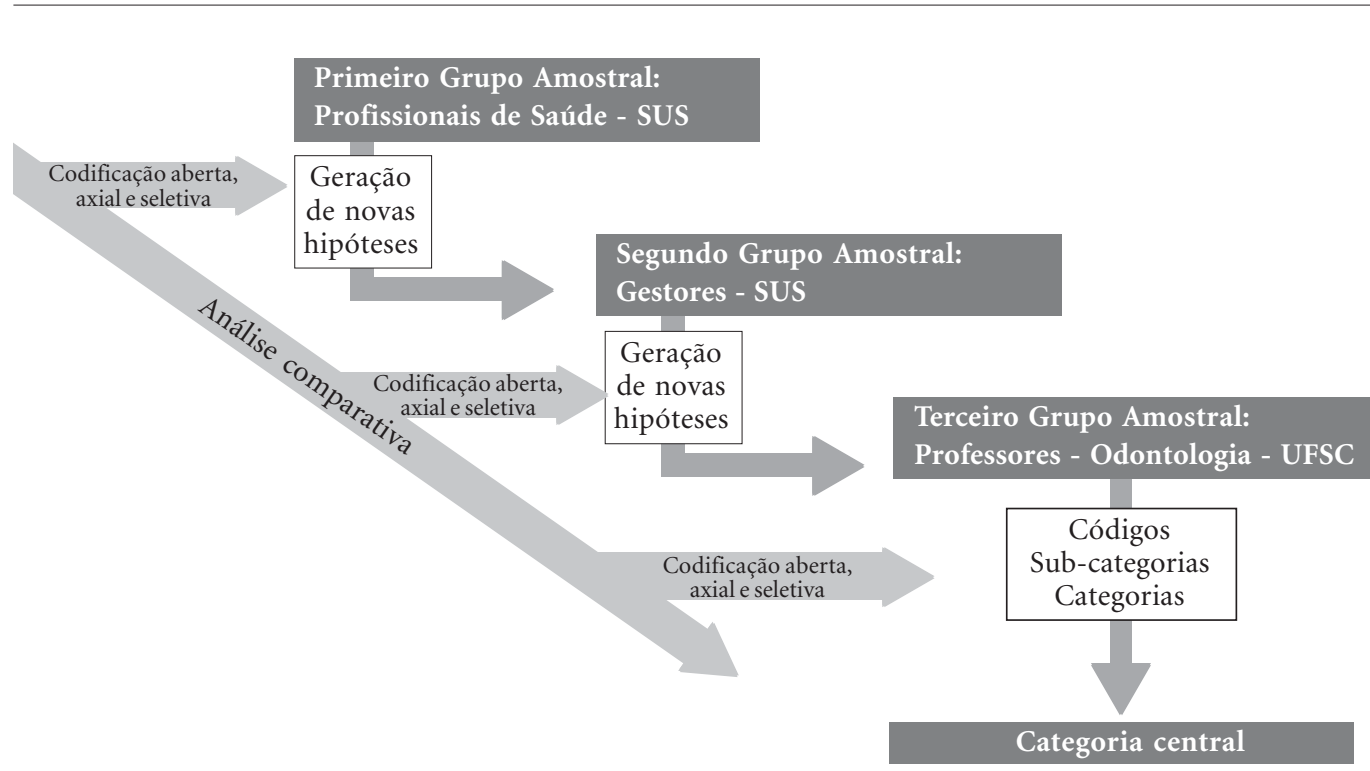

Figura 1. Processo de Amostragem Teórica para definição da composição dos Grupos Amostrais. 
suas propriedades, com a finalidade de alcançar uma Categoria Central abrangente e representativa do modelo teórico.

Este estudo foi aprovado pelo Comitê de Ética em Pesquisa da UFSC. Aos participantes da pesquisa foi assegurado o direito de acesso aos dados e anonimato, bem como foi solicitado consentimento por escrito, atendendo às recomendações do Conselho Nacional de Saúde.

\section{Resultados e Discussão}

A proposta de uma rede de cuidados progressivos, conforme as diretrizes da Política Nacional de Saúde Bucal ${ }^{11}$, delineia uma possível organização da atenção, a partir das áreas de trabalho dos profissionais, da demanda espontânea e de grupos prioritários ${ }^{10}$, considerando os diferentes níveis de atenção em saúde. A Figura 2 mostra o componente da assistência no campo da atenção à Saúde Bucal em um território.

A construção do modelo teórico sobre a inserção da saúde bucal na rede de atenção à saúde, considerando o processo de regionalização, tem por alicerce a categoria central denominada Potencializando a interação na rede de atenção à saúde bucal no processo de regionalização. A identificação desta categoria central resultou da integração de três subcategorias emergentes: a) $\mathrm{Ca}$ racterísticas do cuidado à saúde bucal na rede regionalizada de atenção à saúde; b) Dificuldades na implementação da rede regionalizada de atenção à saúde bucal; e c) O funcionamento da rede de atenção à saúde bucal: realidade e projeções. Este modelo teórico levou à construção de uma representação gráfica (Figura 3) alusiva à realidade encontrada.

\section{Características do cuidado à saúde bucal na rede regionalizada de atenção à saúde}

A inserção do cuidado à saúde bucal na rede regionalizada de atenção à saúde foi caracterizada pelos participantes como um processo ainda em fase inicial, considerado incipiente, por vezes descolado da rede de atenção, que também está por se consolidar.

Medidas político-administrativas nos últimos anos como, por exemplo, a ampliação do acesso à atenção primária, a inserção da ESB na ESF, o foco nas ações coletivas, a definição de linhas de cuidado e os incentivos financeiros, fortalecem a atenção à saúde bucal como uma política pública e promovem sua expansão, qualificação e visibilidade, constituindo fatores de estímulo para a concepção da rede de saúde com componentes bucais $^{10,11}$.

Devido ao recente processo de implementação da rede de atenção à saúde na Região da Grande Florianópolis ${ }^{15}$, a inserção do cuidado à saúde bucal sofre influências motivadas pelas indefinições sobre sua funcionalidade, aspectos estruturais, pontos de produção de ações e serviços, sistemas de apoio, bem como as articulações necessárias entre os componentes para o cumprimento de objetivos.

Há grande discrepância entre os municípios da Região de Saúde da Grande Florianópolis na capacidade de absorver as diretrizes da Política Nacional de Saúde Bucal, e transformá-las em ações executadas na amplitude e profundidade necessárias à melhoria do quadro epidemiológico bucal da população. Vale ressaltar que o município de São José ainda não implantou equipes de saúde bucal na ESF. Apesar de muito desigual entre municípios e mesmo entre UBS de um mes-

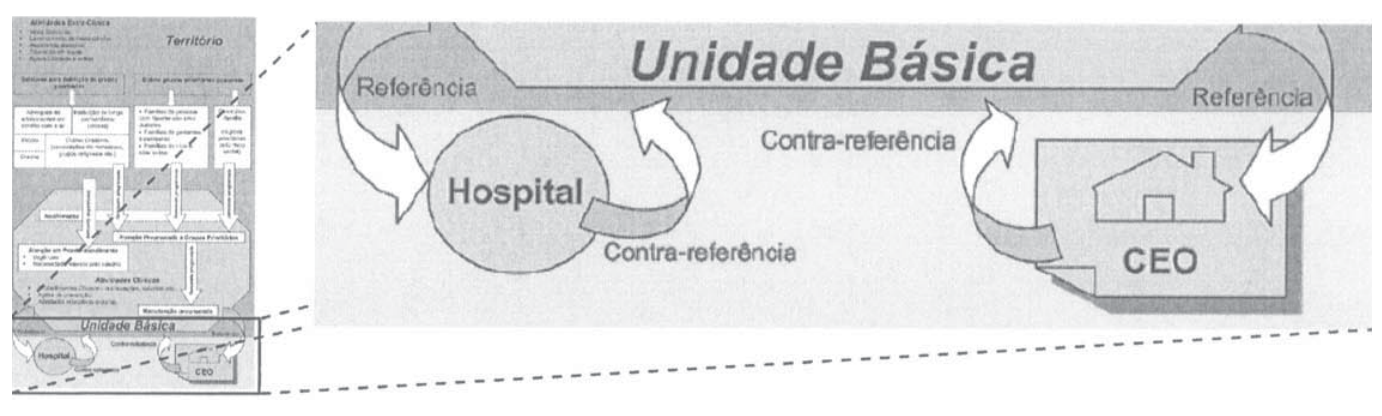

Figura 2. Componente assistencial do campo da atenção à saúde bucal no território. 


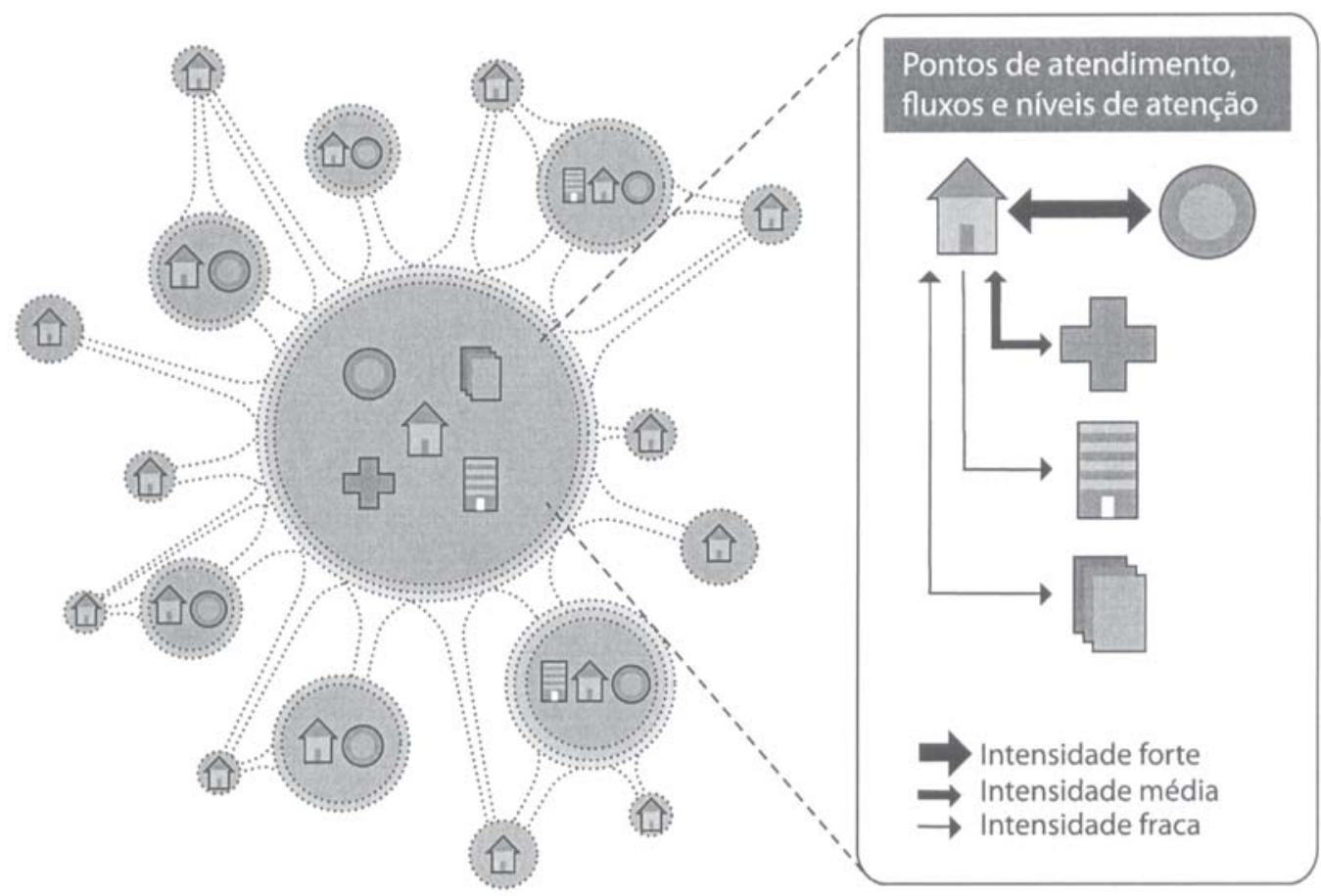

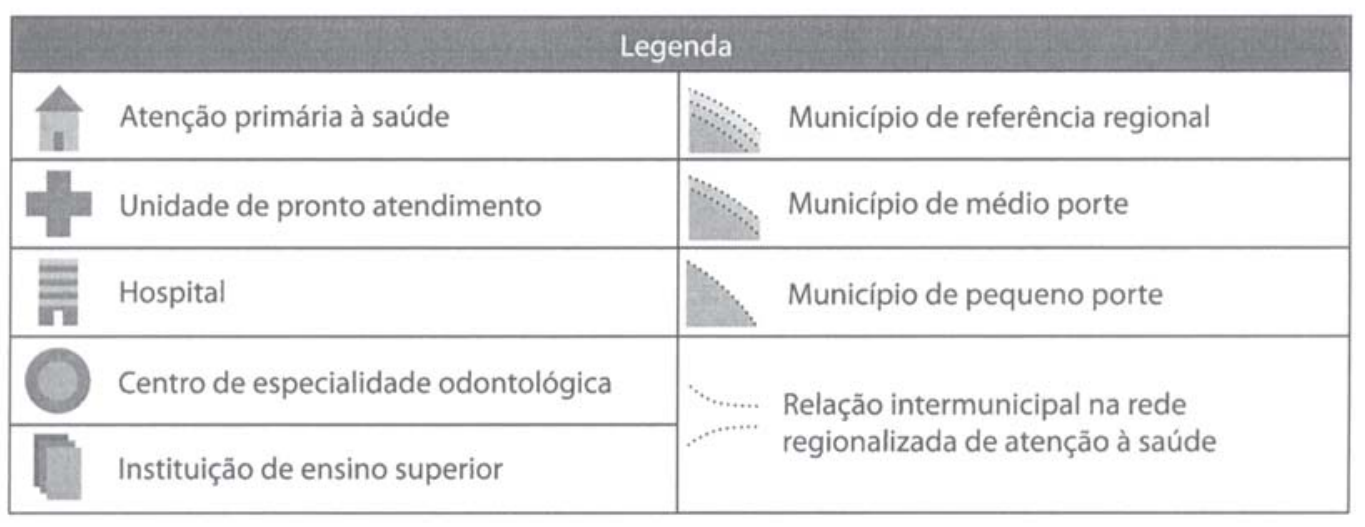

Figura 3. Interação na rede de atenção à saúde bucal no processo de regionalização.

mo município, na visão dos participantes, a saúde bucal na atenção básica tem conseguido evoluir na aplicação dos princípios organizativos da APS, de modo que esta seja a porta de entrada para acesso ao sistema de saúde, que haja responsabilização dos profissionais pelos usuários e criação de vínculo com a comunidade, que o trabalho seja feito por uma equipe multiprofissional, que as ações tenham base territorial.

Segundo os entrevistados, face às necessidades acumuladas pela população quanto à saúde bucal e à presença de uma importante demanda reprimida, os serviços odontológicos na atenção primária são comprimidos pela busca de pronta resposta por parte da população. As ações coletivas ainda permanecem coadjuvantes na inserção do cuidado à saúde bucal na rede de atenção, pois o foco é direcionado às práticas assistenciais, cuja visibilidade do resultado é de curto prazo. Bonfada et al. ${ }^{16}$ constatam que a maior parte dos entraves para a melhoria dos serviços prestados pelo SUS diz respeito ao predomínio do modelo médico assistencial curativista no fazer/ pensar saúde dos profissionais. A clínica tradicional biologicista precisa ser desconstruída, rompendo com a lógica do sofrimento manifesto e da queixa-conduta, com vistas a reorganização tecnológica das ações ${ }^{16}$. 
Há uma percepção de que, embora haja alguma cobrança pela execução de ações coletivas promocionais e preventivas, o interesse e o compromisso do profissional cirurgião-dentista é que, em última análise, determina a sua realização, abrangência, os referenciais teóricos que a sustentam e sua qualidade. As ações interdisciplinares não estão consolidadas de modo que haja discussões mais amplas entre colegas da equipe, permitindo interface da saúde bucal com outras áreas da saúde e do cuidado. $\mathrm{O}$ atendimento das demandas espontâneas, com oferta de procedimentos clínico-curativos, prevalece como garantia mínima de acesso à atenção primária.

A estruturação da média complexidade na Odontologia, em especial pela implantação dos CEO, proporcionou vazão aos procedimentos de maior densidade tecnológica e a continuidade da assistência ao usuário, sem rompimento da linha de cuidado à saúde bucal. Assim, iniciativas municipais isoladas ou conjuntas com a estruturação de um centro de referência, foram consideradas pelos entrevistados marcos para a estruturação de uma rede de atenção à saúde bucal, na qual o usuário tem acesso ao atendimento na atenção primária, com encaminhamento para os centros de especialidade. O processo de distribuição dos CEO na Região da Grande Florianópolis manteve o foco na regionalização, a partir de parâmetros geodemográficos. A inclusão de atendimento odontológico de urgência 24 horas nas Unidades de Pronto Atendimento (UPA) na capital é relevante para a construção da rede de atenção à saúde bucal. Além do escoamento das demandas de urgência/emergência, o serviço oferecido pela UPA favorece o processo de trabalho das ESB com foco na atenção primária.

A UFSC desempenha um papel importante na construção da rede de atenção à saúde bucal, na Região da Grande Florianópolis, com a prestação de serviços odontológicos pelo curso de Odontologia. Os serviços oferecidos absorvem demandas locais e regionais, principalmente no que se refere a atendimento de média e alta complexidade. Cabe recordar que a UFSC e a Prefeitura Municipal de Florianópolis instituíram uma representação colegiada, na qual participam trabalhadores da saúde, estudantes, professores e representantes do Conselho Municipal de Saúde, denominada Rede Docente Assistencial $(\mathrm{RDA})^{17}$. Esta rede define cenários de prática e campos de estágio de cursos e estudantes, bem como os modos de integração nesses locais. Trata-se de um espaço de discussão e gestão que possibilita, também, a integração entre os cursos da área da saúde. Nesse contexto, cabe também ressaltar a instituição dos grupos PET-Saúde, com participação de alunos do curso de graduação em Odontologia em atividades de ensino, pesquisa e extensão na rede do município de Florianópolis. Segundo Souza e Carcereri ${ }^{18}$ a integração ensino-serviço é uma estratégia que potencializa o processo de mudança de práticas na formação em saúde, a partir da reestruturação curricular, por meio de diversas ações na interface do ensino com o serviço. Essas transformações no âmbito da formação são fundamentais para a implementação do trabalho em saúde na perspectiva da atenção em rede ${ }^{18}$.

Uma característica que influencia substantivamente a efetivação da rede em saúde bucal consiste no frágil, senão nulo, conhecimento do sistema pelo usuário, especialmente sobre os pontos de atendimento, os fluxos e os limites de atuação de cada nível de atenção. O estudo de Andrade e Ferreira $^{19}$ revelou, por exemplo, que o trabalho da equipe na ESF não é percebido pelos usuários, que desconhecem a estratégia e a inclusão da odontologia, culminando com uma avaliação negativa do seu atendimento no contexto estudado. Os entrevistados consideram tratar-se de competência do serviço de saúde a realização do acolhimento das pessoas, visto que a orientação interessada, o estímulo à corresponsabilização e à promoção da autonomia do indivíduo são, também, atribuições dos profissionais e gestores da saúde.

Apesar dos esforços e avanços visíveis, os entrevistados percebem que há uma distância entre o previsto na legislação e a realidade vivenciada nos diversos níveis de atenção e nos sistemas de apoio e logísticos da rede, para que o cuidado à saúde bucal se efetive de forma integral.

\section{Dificuldades na implementação da rede regionalizada de atenção à saúde bucal}

Um dos principais motivos que dificultam a plena inserção do cuidado à saúde bucal na rede de atenção à saúde reside na fragilidade do cumprimento das atribuições relativas ao nível de atenção primária. Na condição de estratégia de reorganização do sistema de serviços de saúde ${ }^{20}$, com foco na satisfação das necessidades e demandas da população, o primeiro contato ou o acesso do usuário aos serviços odontológicos, bem como a articulação com outros níveis de atenção são identificados como fragilidade para a garantia de acesso universal.

O planejamento das ações de saúde bucal, a definição de áreas programáticas e projetos, ba- 
seados no levantamento epidemiológico, e a tradução em indicadores não constitui rotina no processo de trabalho das ESB ou do CD. Os dados epidemiológicos em nível municipal são escassos. Florianópolis divulga em seu sitio eletrônico dados resultantes de levantamento epidemiológico municipal da cárie dentária: em 2008, o índice CPO-D atingiu 0,76 aos 12 anos de idade. A literatura tem discutido as razões para o declínio nos índice de cárie dentária, sendo a hipótese mais considerada a elevação no acesso à água e creme dental fluorados e as mudanças nos programas de saúde bucal coletiva ${ }^{21}$. Para a Região de Saúde da Grande Florianópolis, ainda são necessários mais estudos no sentido de especular os reais fatores que influenciam este quadro, nos diferentes cenários municipais.

Outra fragilidade reside no fato do trabalho odontológico predominar em âmbito restrito, de consultório, distanciado de uma compreensão ampliada da base territorial e das determinações socialmente produzidas, nas quais o processo saúde-doença bucal se desenvolve ${ }^{22}$. Ao manter o enfoque assistencialista, os entrevistados questionam até que ponto os serviços odontológicos, do modo como hoje estão posicionados na rede, têm o potencial de reverter o quadro epidemiológico bucal da população e minimizar os efeitos dos determinantes sociais das doenças bucais. $\mathrm{O}$ estudo de Celeste et al. ${ }^{23}$ discutiu a possibilidade dos serviços odontológicos influenciarem a variação na saúde bucal entre indivíduos e entre populações, visto que de forma geral, a influência dos serviços é aparentemente menor do que aquelas relacionadas às condições socioeconômicas. No contexto do seu estudo, o serviço público de odontologia pode ter contribuído para a redução no número de lesões de cárie não restauradas, porém não foi possível detectar influência desse serviço na experiência total da cárie ${ }^{23}$.

A Região de Saúde da Grande Florianópolis concentra um grande número de serviços especializados, de maior densidade tecnológica, tanto para diagnóstico quanto para tratamento odontológico, em comparação a outras regiões do Estado. Para os entrevistados, a rede bucal não está organizada de modo a atender majoritariamente pessoas da Região da Grande Florianópolis, havendo uma sobrecarga em virtude da ausência ou limitação dos serviços em cidades do interior do Estado. A estrutura atual dos serviços de saúde bucal na atenção secundária está aquém das necessidades da população e por isso ainda é insuficiente o número de vagas oferecidas. Além disso, a atenção terciária, em nível hos- pitalar, oferece poucas vagas concentradas nas áreas de estomatologia, trauma, atendimento a portadores de fissuras lábio-palatais e pacientes com necessidades especiais.

Estudo de Chaves e Vieira-da-Silva ${ }^{3}$ investigou a descentralização da gestão dos serviços de saúde bucal, tendo concluído que este processo não vem sendo acompanhado de um esforço deliberado e amplo de melhoria da capacidade administrativa e de gestão dos serviços, apesar do aumento de repasses de recursos financeiros para o setor. Os melhores resultados foram observados onde existia um projeto de reorganização das práticas de saúde, de capacidade técnica e governabilidade 3 . Os participantes reconhecem que as decisões no âmbito da saúde bucal competem ao gestor municipal e que, por conta disso, elas seguem uma orientação muito mais política do que técnica, em geral. As iniciativas/decisões dependem mais de visões pessoais do gestor em exercício do que fruto de um sistema de governança adequado ao SUS.

Mesmo com a ocorrência de novas contratações, a escassez de pessoal - cirurgiões dentistas, auxiliares e técnicos de saúde bucal - foi considerada outra dificuldade para a construção de uma rede que atenda o conjunto de problemas acumulado na população usuária. Adicionalmente, o desconhecimento sobre o processo de trabalho do cirurgião-dentista no SUS traz à tona a falta de protocolos como um potencial prejuízo do trabalho em rede. No âmbito de um mesmo município, os centros de saúde têm práticas e condutas distintas ${ }^{24}$.

A exigência por produtividade, vivenciada por todos os entrevistados, independentemente do ponto no qual se insere na rede, parece ser gerada por uma cadeia de cobranças, desde o nível federal ao local, que valoriza e contabiliza números de procedimentos.

Dificuldades adicionais giram em torno de falta de recursos materiais, em termos de quantidade e qualidade; restrição de espaço físico e de equipamentos; baixa arrecadação financeira em municípios de menor porte; fragilidade do serviço de apoio diagnóstico, com poucos equipamentos de RX; desarticulação no sistema de transporte intermunicipal de usuários; e déficit de registro nos sistemas de informação.

\section{O funcionamento da rede de atenção à saúde bucal: realidade e projeções}

As características desejadas ao pleno funcionamento de uma rede de atenção à saúde bucal, 
apontadas pelos entrevistados, coincidem com muitas das premissas que fundamentam a organização de redes de atenção à saúde, indicadas pelo Ministério da Saúde brasileiro: centralidade no usuário, integração produtiva e continuidade do cuidado, reconhecimento da complementaridade e da interdependência, construção de objetivos comuns e racionalidade sistêmica, coordenação, flexibilidade e desempenho, compartilhamento decisório, responsabilização e institucionalidade, pluralidade, democratização e transparência ${ }^{9,10}$.

O cuidado à saúde bucal deve ocupar espaço nas discussões sobre a estruturação das redes regionalizadas de atenção à saúde. Para isso é necessária a participação de seus profissionais, gestores ou não, nas instâncias que agregam poder decisório. Alguns protocolos operacionais à funcionalidade da rede, como de referência e contrarreferência, de padronização de materiais e instrumentais odontológicos, de terapêutica, de atendimento em urgência e emergência/acolhimento, de atribuições de técnicos e auxiliares de saúde bucal, foram citados pelos participantes. A elaboração desses protocolos deve ser uma construção coletiva, conjugando interesses de gestores e profissionais, para que não engessem o processo de trabalho.

O funcionamento da rede requer o reconhecimento dos seus pontos de atenção e fluxos por parte dos profissionais da saúde bucal (cirurgião-dentista, técnicos e auxiliares) de modo que a utilizem adequadamente, aproveitando ao máximo a oferta de serviços tão caros ao usuário. A lacuna de conhecimento por parte dos profissionais precisa ser superada via estímulo e operacionalização da educação permanente. Esta, por sua vez, deve ser expandida, com a finalidade de subsidiar municípios de menor porte, distantes das instituições formadoras tradicionais.

Os participantes indicaram a importância da interação entre os diversos níveis constituintes da rede, bem como das relações intersetoriais para dialogar, refletir e agir articuladamente sobre a situação da saúde bucal, em nível local e regional. Essas discussões devem também alcançar espaços de participação popular de modo a promover a sensibilização da comunidade ou seus representantes, sobre a saúde bucal no SUS.

A UFSC e o Curso de Graduação em Odontologia foram identificados pelos entrevistados como parte integrante da rede de atenção à saúde bucal. Consideraram que sua participação, bem como as contribuições daí advindas podem ser potencializadas de modo a promover a qualificação desta rede. O redirecionamento no modo de organizar as ações de cuidado da saúde bucal no Brasil implica necessariamente a integração com instituições formadoras, referências na produção de conhecimento científico e desenvolvimento de tecnologias na área da Odontologia e saúde bucal. O cenário de transformação apresenta, para as universidades, a desafiadora tarefa de implementar diretrizes curriculares coerentes com as demandas políticas e sociais ${ }^{25}$. Para os entrevistados, o currículo deve estar construído de forma a responder às necessidades da população, e em função dos problemas de saúde bucal mais prevalentes ${ }^{18,26}$.

A formação técnica do cirurgião-dentista foi considerada tradicional, acrítica, pouco reflexiva, segmentada, voltada para a inserção no mercado privado, com foco no atendimento curativo e individualizado. Esta formação não possui aderência à realidade brasileira, tanto em termos epidemiológicos quanto em relação ao modelo de atenção à saúde pretendido no âmbito do SUS. Todavia, reconhecem o esforço que vem sendo realizado para modificar esta realidade, que pode ser atestado por algumas publicações com foco na reorientação da formação do profissional de saúde no Brasil25-27.

Embora os entrevistados avaliem como reduzido o quantitativo de cirurgiões dentistas para a cobertura da demanda, há um reconhecimento de que, nos últimos anos, o SUS tem ofertado maior número de vagas para cirurgião-dentista e ampliado a inserção de profissionais da equipe de saúde bucal na ESF, incluindo os técnicos e auxiliares. A ocupação destes postos de trabalho vem se dando por profissionais recém-formados, nem sempre com qualificação específica para muitas das atribuições exigidas pelo sistema público de saúde.

A funcionalidade da rede de atenção à saúde bucal vincula-se à assimilação de, pelo menos, três premissas, ainda em processo de consolidação no campo da saúde. A primeira relativa à saúde bucal como parte integrante e inseparável da saúde e componente que influencia o bemestar das pessoas; a segunda diz respeito à valorização da Odontologia, enquanto profissão que se preocupa com as questões relativas ao processo saúde-doença bucal na população; e, por último, a definitiva inserção dos profissionais da saúde bucal, na condição de integrantes do sistema público, em equipe de saúde que trabalha sob bases interdisciplinares. 


\section{Considerações finais}

Os elementos recolhidos neste estudo indicam a implantação da rede regionalizada de atenção à saúde como condição para levar o cuidado à saúde bucal a um novo patamar de atenção e assistência. Aí residem as contribuições trazidas pela construção deste modelo teórico sobre a inserção da saúde bucal na rede de atenção à saúde, que considera o processo de regionalização e aponta caminhos para a potencialização das interações nessa rede.

A pesquisa mostrou que a atuação fracionada e autárquica da organização das ações e serviços de saúde bucal, que prevaleceu até recentemente, é vista com desconforto e como padrão a ser rejeitado na proposta da estruturação da Rede de Atenção à Saúde. Desde logo, especula-se a oportunidade do rompimento do isolamento da saúde bucal e da Odontologia, pela participação de uma ação regional mais cooperativa e integrada na rede.

Em relação ao fortalecimento da atenção primária, especificamente, isto significa mais do que a ampliação do acesso. Reforça a transformação deste nível de atenção em uma estratégia de organização do sistema, reordenando os recursos disponíveis aos usuários. A adoção da ESF como mecanismo de reorientação das ações de saúde bucal na atenção primária é requisito para composição da rede, pela implantação de equipes de saúde bucal com boa cobertura nos municípios. A partir da (re)estruturação do atendimento da atenção primária, progressivamente vão se traçando os contornos dos serviços da rede de atenção secundária e terciária. A formalização de fluxos entre os pontos de atenção conduzirá a uma plena interação da saúde bucal na RAS, na região estudada.

\section{Colaboradores}

ALSF Mello participou da concepção do estudo, coleta e análise dos dados e redação do manuscrito. SR Andrade, SJ Moysés e AL Erdmann participaram da análise dos dados e redação do manuscrito. 


\section{Referências}

1. Souza RR. A regionalização no contexto atual das políticas de saúde. Cien Saude Colet 2001; 6(2);451455.

2. Pasche DF, Righi LB, Thome HI, Stolz ED. Paradoxos das políticas de descentralização de saúde no Brasil. Rev Panam Salud Publica. 2006; 20(6):416422.

3. Chaves SCL, Vieira-da-Silva LM. Atenção à saúde bucal e a descentralização da saúde no Brasil: estudo de dois casos exemplares no Estado da Bahia Cad Saude Publica 2007; 23(5):1119-1131.

4. Silva SF, organizador. Redes de atenção à saúde no SUS: o pacto pela saúde e redes regionalizadas de ações e serviços de saúde. Campinas: IDISA; 2008.

5. Mendes EV. As redes de atenção à saúde. Cien Saude Colet 2010;15(5):2297-05.

6. Pan American Health Organization (PAHO). Renewing primary health care in the Americas: a position paper of the Pan American Health Organization/World Health Organization. Washington PAHO/ WHO; 2007.

7. Organización Panamericana de la Salud (OPAS). Redes integradas de servicios de salud: conceptos, opciones de política y hoja de ruta para su implementación en las Américas. Washington: OPAS; 2010. (Serie: La Renovación de la Atención Primaria de Salud en las Américas, No 4).

8. Vázquez ML, Vargas I, Unger JP, Mogollón A, Silva MRF, de Paepe P. Integrated health care networks in Latin America: toward a conceptual framework for analysis. Rev Panam Salud Publica 2009; 26(4): 360-367.

9. Brasil. Portaria 4.279, de 30 de dezembro de 2010 . Estabelece diretrizes para a organização da Rede de Atenção à Saúde no âmbito do Sistema Único de Saúde (SUS). Diário Oficial da União 2010; 31 dez.

10. Brasil. Ministério da Saúde (MS). Secretaria de Atenção à Saúde. Departamento de Atenção Básica. Cadernos de atenção básica. Saúde Bucal no 17. Brasília: MS; 2006.

11. Brasil. Ministério da Saúde (MS). Secretaria de Atenção à Saúde. Departamento de Atenção Básica. Coordenação Nacional de Saúde Bucal. Diretrizes da Política Nacional de Saúde Bucal. Brasília: MS; 2004.

12. Charmaz K. Constructing grounded theory: a practical guide through qualitative analysis. London: Sage Publications; 2007.

13. Glaser B. Grounded theory perspective III: theoretical coding. Chicago: Sociology Press; 2005.

14. Brasil. Departamento de Informática do SUS (DATASUS). Sistema de Informação em Saúde. [página na Internet]. [acessado 2012 maio 7]. Disponível em: http://www2.datasus.gov.br/DATASUS/index. php?area $=0203$

15. Santa Catarina. Secretaria de Estado da Saúde (SES) Plano Diretor de Regionalização - 2008. Florianópolis: SES; 2008.

16. Bonfada D, Cavalcante JRLP, Araújo DP, Guimarães $\mathrm{J}$. A integralidade da atenção à saúde como eixo da organização tecnológica nos serviços. Cien Saude Colet 2012; 17(2):555-560.
17. Universidade Federal de Santa Catarina (UFSC). Hospital Universitário Secretaria da Saúde de Florianópolis. Regimento interno da rede de articulação. Universidade - Sistema de Saúde/Rede Docente Assistencial de Florianópolis. Florianópolis: UFSC; 2006.

18. Souza AL, Carcereri DL. Estudo qualitativo da integração ensino-serviço em um curso de graduação em Odontologia. Interface (Botucatu). 2011; 15(3): 1071-1084.

19. Andrade KLC, Ferreira EF. Avaliação da inserção da odontologia no Programa Saúde da Família de Pompéu (MG): a satisfação do usuário. Cien Saude Colet 2006; 11(1):123-130.

20. Brasil, Ministério da Saúde (MS). Portaria no 648/ GM, de 28 de março de 2006. Aprova a Política Nacional de Atenção Básica. Diário Oficial da União 2006; 29 mar.

21. Narvai PC, Frazão P, Roncalli AG, Antunes JLF. Cárie dentária no Brasil: declínio, iniqüidade e exclusão social. Rev Panam Salud Publica 2006; 19(6):385-393.

22. Sheiham A, Alexander D, Cohen L, Marinho V, Moysés S, Petersen PE, Spencer J, Watt RG, Weyant R. Global oral health inequalities: task group - implementation and delivery of oral health strategies. Adv Dent Res 2011; 23(2):259-267.

23. Celeste RK, Nadanovsky P, Leon AP. Associação entre procedimentos preventivos no serviço público de odontologia e a prevalência de cárie dentária. Rev Saude Publica 2007: 41(5):830-838.

24. Santos APPD, Nadanovsky P, Oliveira BHD. Pesquisa com Secretarias de Saúde no Brasil revela recomendações divergentes relacionadas a práticas de higiene bucal em crianças. Cad Saude Publica 2010; 26(7):1457-1463.

25. Brasil. Resolução CNE/CES no 2, de 19 de fevereiro de 2002. Institui as diretrizes curriculares nacionais dos cursos de graduação em Farmácia e Odontologia. Diário Oficial da União 2002; 20 fev.

26. Freitas SFT, Calvo, MCM, Lacerda JT. Saúde coletiva e novas diretrizes curriculares em odontologia: uma proposta para graduação. Trab. educ. saúde. 2012: 10(2):223-234

27. Brasil, Ministério da Saúde (MS). Programa nacional de reorientação da formação profissional em saúde (PRÓ-SAÚDE). Portaria Interministeria 2.101, de 3 de novembro de 2001. Institui o Programa Nacional de Reorientação da Formação Profissional em Saúde - Pró-Saúde - para os cursos de graduação em Medicina, Enfermagem e Odontologia. Diário Oficial da União 2005; 4 nov.

Artigo apresentado em 23/07/2012

Aprovado em 07/09/2012

Versão final apresentada em 21/09/2012 